\title{
On a DGL-map between derivations of Sullivan minimal models
}

Received: 6 May 2020 / Accepted: 4 August 2020 / Published online: 18 August 2020

(C) The Author(s) 2020

\begin{abstract}
For a map $f: X \rightarrow Y$, there is the relative model $M(Y)=(\Lambda V, d) \rightarrow(\Lambda V \otimes \Lambda W, D) \simeq M(X)$ by Sullivan model theory (Félix et al., Rational homotopy theory, graduate texts in mathematics, Springer, Berlin, 2007). Let Baut $X$ be the Dold-Lashof classifying space of orientable fibrations with fiber $X$ (Dold and Lashof, Ill J Math 3:285-305, 1959]). Its DGL (differential graded Lie algebra)-model is given by the derivations $\operatorname{Der} M(X)$ of the Sullivan minimal model $M(X)$ of $X$. Then we consider the condition that the restriction $b_{f}: \operatorname{Der}(\Lambda V \otimes \Lambda W, D) \rightarrow \operatorname{Der}(\Lambda V, d)$ is a DGL-map and the related topics.
\end{abstract}

\section{Mathematics Subject Classification $55 \mathrm{P} 62 \cdot 55 \mathrm{R} 15$}

\section{Introduction}

Let $X$ (and also $Y$ ) be a connected and simply connected finite CW complex with $\operatorname{dim} \pi_{*}(X)_{\mathbb{Q}}<\infty$ $\left(G_{\mathbb{Q}}=G \otimes \mathbb{Q}\right)$ and Baut $_{1} X$ be the Dold-Lashof classifying space of orientable fibrations [5]. Here aut $_{1} X=\operatorname{map}\left(X, X ; i d_{X}\right)$ is the identity component of the space aut $X$ of self-equivalences of $X$. Then any orientable fibration $\xi$ with fibre $X$ over a base space $B$ is the pull-back of a universal fibration $X \rightarrow E_{\infty}^{X} \rightarrow$ Baut $_{1} X$ by a map $h: B \rightarrow$ Baut $_{1} X$ and equivalence classes of $\xi$ are classified by their homotopy classes [2,5,23]. The Sullivan minimal model $M(X)$ [24] determines the rational homotopy type of $X$, the homotopy type of the rationalization $X_{0}$ [14] of $X$. Notice that $\left(\operatorname{Baut}_{1} X\right)_{0} \simeq \operatorname{Baut}_{1}\left(X_{0}\right)$ [17]. The differential graded Lie algebra (DGL) Der $M(X)$, the negative derivations of $M(X)$ (see $\S 2$ ), gives rise to the DGL model for Baut ${ }_{1} X$ due to Sullivan [24] (cf.[10,25]), i.e., the spatial realization $\|\operatorname{Der} M(X)\|$ is $\left(\operatorname{Baut}_{1} X\right)_{0}$. Therefore, we obtain a map $\left(\text { Baut }_{1} X\right)_{0} \rightarrow\left(\operatorname{Baut}_{1} Y\right)_{0}$ if there is a DGL-map Der $M(X) \rightarrow \operatorname{Der} M(Y)$. However, it does not exist in general.

Let $f: X \rightarrow Y$ be a map whose homotopy fibration $\xi_{f}: F_{f} \rightarrow X \rightarrow Y$ is given by the relative model (Koszul-Sullivan extension)

$$
M(Y)=(\Lambda V, d) \stackrel{i}{\hookrightarrow}(\Lambda V \otimes \Lambda W, D) \simeq M(X)
$$

for a certain differential $D$ with $\left.D\right|_{\Lambda V}=d$, where $M\left(F_{f}\right) \cong(\Lambda W, \bar{D})$ for the homotopy fiber $F_{f}$ of $f$ [7]. In this paper, we propose

Question 1.1 When is the restriction map given by $b_{f}(\sigma)=\operatorname{proj}_{V} \circ \sigma \circ i$

$$
b_{f}: \operatorname{Der}(\Lambda V \otimes \Lambda W, D) \rightarrow \operatorname{Der}(\Lambda V, d)
$$

a DGL-map ?

T. Yamaguchi

Kochi University, 2-5-1, Kochi 780-8520, Japan

E-mail: tyamag@kochi-u.ac.jp 
Here $\operatorname{proj}_{V}: \Lambda V \otimes \Lambda W \rightarrow \Lambda V$ is the algebra map with $\operatorname{proj}_{V}(w)=0$ for $w \in W$ and $\left.\operatorname{proj}_{V}\right|_{\Lambda V}=i d_{\Lambda V}$.

Definition 1.2 We say that a $\mathbb{Q}$-w.t. map $f: X \rightarrow Y$ strictly induces the map

$$
a_{f}:\left(\text { Baut }_{1} X\right)_{0} \rightarrow\left(\operatorname{Baut}_{1} Y\right)_{0}
$$

if its DGL model is given by the DGL-map $b_{f}: \operatorname{Der}(\Lambda V \otimes \Lambda W, D) \rightarrow \operatorname{Der}(\Lambda V, d)$ with $\left\|b_{f}\right\|=a_{f}$.

Let $\min \pi_{*}(S)_{\mathbb{Q}}:=\min \left\{i>0 \mid \pi_{i}(S)_{\mathbb{Q}} \neq 0\right\}$ and $\max \pi_{*}(S)_{\mathbb{Q}}:=\max \left\{i \geq 0 \mid \pi_{i}(S)_{\mathbb{Q}} \neq 0\right\}$ for a space $S$. In particular, $\min \pi_{*}(S)_{\mathbb{Q}}:=\infty$ when $S$ is the one point space.

Definition 1.3 A fibration $\xi_{f}: F_{f} \rightarrow X \stackrel{f}{\rightarrow} Y$ or a map $f: X \rightarrow Y$ with homotopy fiber $F_{f}$ is said to be $\pi_{\mathbb{Q}}$-separable if $\min \pi_{*}\left(F_{f}\right)_{\mathbb{Q}} \geq \max \pi_{*}(Y)_{\mathbb{Q}}$.

We say a map is rationally weakly trivial (abbr., $\mathbb{Q}$-w.t.) if $\xi_{f}$ is rationally weakly trivial, i.e., $\pi_{*}(X)_{\mathbb{Q}}=$ $\pi_{*}\left(F_{f}\right)_{\mathbb{Q}} \oplus \pi_{*}(Y)_{\mathbb{Q}}$. Then $(\Lambda V \otimes \Lambda W, D)$ is just the minimal model $M(X)$ of $X$. If a map $f: X \rightarrow Y$ is $\pi_{\mathbb{Q}}$-separable, it is $\mathbb{Q}$-w.t. The condition to be $\pi_{\mathbb{Q}}$-separable is equivalent to the condition that $\min W=$ $\min \left\{i>0 \mid W^{i} \neq 0\right\} \geq \max V=\max \left\{i>0 \mid V^{i} \neq 0\right\}$ in the relative minimal model $M(Y)=(\Lambda V, d) \rightarrow$ $(\Lambda V \otimes \Lambda W, D)$ of $\xi_{f}$.

Proposition 1.4 For $a \mathbb{Q}$-w.t. map $f: X \rightarrow Y$ with relative model $M(Y)=(\Lambda V, d) \rightarrow(\Lambda V \otimes \Lambda W, D)$, the restriction $b_{f}: \operatorname{Der}(\Lambda V \otimes \Lambda W, D) \rightarrow \operatorname{Der}(\Lambda V, d)$ is a DGL-map if and only if $f$ is $\pi_{\mathbb{Q}}$-separable.

That means

Theorem 1.5 A $\mathbb{Q}$-w.t. map $f: X \rightarrow Y$ strictly induces $a_{f}:\left(\operatorname{Baut}_{1} X\right)_{0} \rightarrow\left(\operatorname{Baut}_{1} Y\right)_{0}$ if and only if $f$ is $\pi_{\mathbb{Q}}$-separable.

In §2, we give the proofs under some preparations of models of [7] and [25]. In this paper, we consider only $\mathbb{Q}$-w.t. maps. For example, we do not consider the inclusion map $i_{X}: X \rightarrow X \times Y$, which is not $\mathbb{Q}$-w.t. However $i_{X}$ induces the monoid map $\psi:$ aut $_{1} X \rightarrow$ aut $_{1}(X \times Y)$ by $\psi(g)=g \times 1_{Y}$ and, therefore, there exists the induced map $B \psi:$ Baut $_{1} X \rightarrow B$ aut $_{1}(X \times Y)$ without rationalization. The DGL model is given by the natural inclusion $\operatorname{Der} M(X) \rightarrow \operatorname{Der}(M(X) \otimes M(Y))$, which is a DGL-map.

In $\S 3$, we give some conditions that the strictly induced map $a_{f}:\left(\operatorname{Baut}_{1} X\right)_{0} \rightarrow\left(\operatorname{Baut}_{1} Y\right)_{0}$ admits a section. Some results are obtained by Proposition 3.1 induced from [25, VI .1.(3) Proposition] that the DGL-model of the homotopy fibration $\chi_{f}: F_{a_{f}} \rightarrow\left(\operatorname{Baut}_{1} X\right)_{0} \stackrel{a_{f}}{\rightarrow}\left(\operatorname{Baut}_{1} Y\right)_{0}$ is given by

$$
\operatorname{Der}(\Lambda W, \Lambda V \otimes \Lambda W) \rightarrow \operatorname{Der}(\Lambda V \otimes \Lambda W) \stackrel{b_{f}}{\rightarrow} \operatorname{Der}(\Lambda V)
$$

Let $\operatorname{aut}_{1} f$ be the identity component of the space of all fibre-homotopy self-equivalences of $f$, i.e., $\{g$ : $X \rightarrow X \mid f \circ g=f\}$ and $\mathrm{Baut}_{1} f$ be the classifying space of this topological monoid. It is known that Baut $_{1} f \simeq \operatorname{map}\left(Y\right.$, Baut $\left._{1}\left(F_{f}\right) ; h\right)$, where $h: Y \rightarrow$ Baut $_{1}\left(F_{f}\right)$ is the classifying map of the fibration $F_{f} \rightarrow X \stackrel{f}{\rightarrow} Y$ and $\widetilde{\text { map }}$ denotes the universal cover of the function space [3]. Notice that

$$
\operatorname{Der}(\Lambda W, \Lambda V \otimes \Lambda W)=\operatorname{Der}_{\Lambda V}(\Lambda V \otimes \Lambda W),
$$

where $\operatorname{Der}_{\Lambda V}(\Lambda V \otimes \Lambda W)$ is the sub DGL of $\operatorname{Der}(\Lambda V \otimes \Lambda W)$ sending the elements of $\Lambda V$ to zero and it is a DGL model of Baut $f f$ when $Y$ and $F_{f}$ are finite [4, Theorem 1], [8]. Thus, we note

Theorem 1.6 If the homotopy fiber $F_{f}$ is finite for a $\pi_{\mathbb{Q}}$-separable map $f$, the fiber of $\chi_{f}$ has the rational homotopy type of Baut $_{1} f$.

A space $X$ is said to be elliptic if the dimensions of the rational cohomology algebra and homotopy group are both finite [7]. An elliptic space $X$ is said to be pure if $d M(X)^{\text {even }}=0$ and $d M(X)^{\text {odd }} \subset M(X)^{\prime}$ rmeven . A pure space is said to be an $F_{0}$-space (or positively elliptic) if $\operatorname{dim} \pi_{\text {even }}(X) \otimes \mathbb{Q}=\operatorname{dim} \pi_{\text {odd }}(X) \otimes \mathbb{Q}$ and $H^{\text {odd }}(X ; \mathbb{Q})=0$. Then it is equivalent to $H^{*}(X ; \mathbb{Q}) \cong \mathbb{Q}\left[x_{1}, \ldots, x_{n}\right] /\left(f_{1}, \ldots, f_{n}\right)$, in which $\left|x_{i}\right|$, the degree of $x_{i}$, is even and $f_{1}, \ldots, f_{n}$ forms a regular sequence in the $\mathbb{Q}$-polynomial algebra $\mathbb{Q}\left[x_{1}, \ldots, x_{n}\right]$, where $M(X)=\left(\mathbb{Q}\left[x_{1}, \ldots, x_{n}\right] \otimes \Lambda\left(y_{1}, \ldots, y_{n}\right), d\right)$ with $d x_{i}=0$ and $d y_{i}=f_{i}$. In 1976, S. Halperin [12] conjectured that the Serre spectral sequences of all fibrations $X \rightarrow E \rightarrow B$ of simply connected CW complexes collapse at the $E_{2}$-terms for any $F_{0}$-space $X$ [7]. For compact connected Lie groups $G$ and $H$ where $H$ is a subgroup of $G$, when rank $G=\operatorname{rank} H$, the homogeneous space $G / H$ satisfies the Halperin conjecture [21]. Also the Halperin conjecture is true when $n \leq 3$ [16]. Finally, we note some relations with the Halperin conjecture $[7, \S 39]$ due to Meier [18] as 
Theorem 1.7 Let $Y$ be an $F_{0}$-space. Then the fibration $\chi_{f}$ is fibre-trivial for any $\pi_{\mathbb{Q}}$-separable map $f: X \rightarrow Y$ if and only if $Y$ satisfies the Halperin's conjecture.

In $\S 4$, we observe the cellular obstruction for the lifting $\tilde{h}$ for a map $h: B \rightarrow\left(\operatorname{Baut}_{1} Y\right)_{0}$ for a simply connected $\mathrm{CW}$ complex $B$ of finite type:

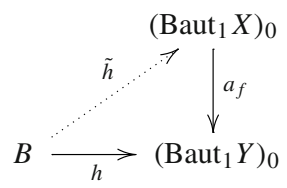

for a $\pi_{\mathbb{Q}^{-}}$-separable map $f: X \rightarrow Y$. Of course, it is sufficient to define as $\tilde{h}=s \circ h$ if $a_{f}$ admits a section $s$. Specifically, for a $\pi_{\mathbb{Q}}$-separable map $f: X \rightarrow Y$, let

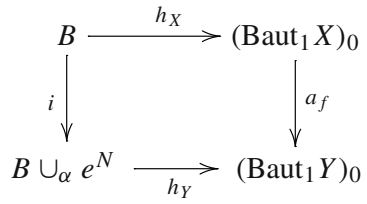

be a commutative diagram. Then, from Proposition 1.6, we define an obstruction class by derivations in Theorem 4.1 so that

Theorem 1.8 Let $f: X \rightarrow Y$ be a $\pi_{\mathbb{Q}}$-separable map with $Y$ and $F_{f}$ finite. There is a lift $h$ such that

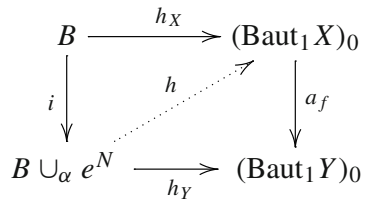

is commutative if and only if $\mathcal{O}_{\alpha}\left(h_{X}, h_{Y}\right)=0$ in $\pi_{N-1}\left(\text { Baut }_{1} f\right)_{\mathbb{Q}}$.

In $\S 5$, we consider an application to lifting actions. Let $G$ be a topological group and acts on a CW complex $Y$. Recall the problem of lifting (up to homotopy) of Gottlieb [11]:

Problem 1.9 When is a fibration $F_{f} \rightarrow X \stackrel{f}{\rightarrow} Y$ fibre homotopy equivalent to a G-fibration? i.e., when is there a fibration $f^{\prime}: X^{\prime} \rightarrow Y$ such that $f^{\prime}$ is fibre homotopy equivalent to $f$ and there is a G-action on $X^{\prime}$ such that $f^{\prime}$ is equivariant?

Suppose that $G$ is a compact connected Lie group. Since $H^{*}(B G ; \mathbb{Q})$ is evenly graded, the obstruction classes of Theorem 1.8 are contained in $\pi_{\text {odd }}\left(\text { Baut }_{1} f\right)_{\mathbb{Q}}$ when $B=B G$. If $\pi_{\text {odd }}\left(\text { Baut }_{1} f\right)_{\mathbb{Q}}=0$, they vanish and there exists a lift $h: B G \rightarrow\left(\text { Baut }_{1} X\right)_{0}$. Then from Theorem 2.6 in the case that $B=B^{\prime}=B G$ and $g=\left(i d_{B G}\right)_{0}$, we obtain using Theorem 5.1 of D. H. Gottlieb.

Theorem 1.10 Let $f: X \rightarrow Y$ be a $\pi_{\mathbb{Q}}$-separable map with $Y$ and $F_{f}$ finite. Suppose that a compact Lie group $G$ acts on $Y$. If $\pi_{\text {odd }}\left(\text { Baut }_{1} f\right)_{\mathbb{Q}}=0$, the action on $Y$ is rationally lifted to $X$, i.e., $f$ is rationally fibre homotopy equivalent to a $G$-equivariant map $f^{\prime}: X^{\prime} \rightarrow Y$ for a $G$-space $X^{\prime}$.

Due to Theorem 1.7 and the result of Shiga and Tezuka [21], we have

Corollary 1.11 Let $f: X \rightarrow Y$ be a $\pi_{\mathbb{Q}}$-separable map such that $Y$ is a homogeneous space $G / H$ with rank $G=\operatorname{rank} H$. Then any group action on $Y$ is rationally lifted to $X$. In particular, the natural $G$-action on $Y$ is rationally lifted to $X$.

Furthermore, we apply the obstruction argument to a rational homotopical invariant: let $r_{0}(X)$ be the rational toral rank of a simply connected complex $X$ of $\operatorname{dim} H^{*}(X ; \mathbb{Q})<\infty$, i.e., the largest integer $r$ such that an $r$-torus $T^{r}=S^{1} \times \cdots \times S^{1}$ (r-factors) can act continuously on a CW-complex $X^{\prime}$ in the rational homotopy type of $X$ with all its isotropy subgroups finite (almost free action) $[1,9,13]$. It is very difficult to calculate $r_{0}()$ in general. From the definition, we have the inequality $r_{0}(X \times Y) \geq r_{0}(X)+r_{0}(Y)$. Notice that it may sometimes be a strict inequality since there is an example that $r_{0}\left(X \times S^{12}\right)>0$ even though $r_{0}(X)=r_{0}\left(S^{12}\right)=0[15$, Example 3.3]. For a map $f: X \rightarrow Y$, we see $r_{0}(Y) \leq r_{0}(X)$ when $X=F \times Y$ for any space $F$ and $f$ is the projection $F \times Y \rightarrow Y$. In general, when does a map $f: X \rightarrow Y$ induce $r_{0}(Y) \leq r_{0}(X)$ ? 
Corollary 1.12 Let $f: X \rightarrow Y$ be a $\pi_{\mathbb{Q}}$-separable map with $Y$ and $F_{f}$ finite. If $\pi_{\text {odd }}\left(\text { Baut }_{1} f\right)_{\mathbb{Q}}=0$, we have $r_{0}(Y) \leq r_{0}(X)$

\section{Sullivan models and derivations}

Let $M(X)=(\Lambda V, d)$ be the Sullivan minimal model of simply connected CW complex $X$ of finite type [24]. It is a free $\mathbb{Q}$-commutative differential graded algebra (DGA) with a $\mathbb{Q}$-graded vector space $V=\bigoplus_{i>1} V^{i}$ where $\operatorname{dim} V^{i}<\infty$ and a decomposable differential, i.e., $d\left(V^{i}\right) \subset\left(\Lambda^{+} V \cdot \Lambda^{+} V\right)^{i+1}$ and $d \circ d=0$. Here $\Lambda^{+} V$ is the ideal of $\Lambda V$ generated by elements of positive degree. The degree of a homogeneous element $x$ of a graded algebra is denoted as $|x|$. Then $x y=(-1)^{|x||y|} y x$ and $d(x y)=d(x) y+(-1)^{|x|} x d(y)$. Note that $M(X)$ determines the rational homotopy type of $X$, namely the spatial realization is given as $\|M(X)\| \simeq X_{0}$. In particular,

$$
V^{n} \cong \operatorname{Hom}\left(\pi_{n}(X), \mathbb{Q}\right) \text { and } H^{*}(\Lambda V, d) \cong H^{*}(X ; \mathbb{Q}) .
$$

Here the second is an isomorphism as graded algebras. Refer to [7] for detail.

Let $\operatorname{Der}_{i} M(X)$ be the set of $\mathbb{Q}$-derivations of $M(X)$ decreasing the degree by $i$ with $\sigma(x y)=\sigma(x) y+$ $(-1)^{i \cdot|x|} x \sigma(y)$ for $x, y \in M(X)$. The boundary operator $\partial: \operatorname{Der}_{i} M(X) \rightarrow \operatorname{Der}_{i-1} M(X)$ is defined by

$$
\partial(\sigma)=d \circ \sigma-(-1)^{i} \sigma \circ d
$$

for $\sigma \in \operatorname{Der}_{i} M(X)$. We denote $\oplus_{i>0} \operatorname{Der}_{i} M(X)$ by $\operatorname{Der} M(X)$ in which $\operatorname{Der}_{1} M(X)$ is $\partial$-cycles. Then $\operatorname{Der} M(X)$ is a (non-free) DGL by the Lie bracket

$$
[\sigma, \tau]:=\sigma \circ \tau-(-1)^{|\sigma||\tau|} \tau \circ \sigma .
$$

Note that $H_{*}(\operatorname{Der} M)=H_{*}(\operatorname{Der} N)$ when free DGAs $M$ and $N$ are quasi-isomorphic [20]. Furthermore, recall the definition of Tanré [25, p. 25]: let $(L, \partial)$ be a DGL of finite type with positive degree. Then $C^{*}(L, \partial)=\left(\Lambda s^{-1} \sharp L, D=d_{1}+d_{2}\right)$ with

$$
\left\langle d_{1} s^{-1} z ; s x\right\rangle=-\langle z ; \partial x\rangle \text { and }\left\langle d_{2} s^{-1} z ; s x_{1}, s x_{2}\right\rangle=(-1)^{\left|x_{1}\right|}\left\langle z ;\left[x_{1}, x_{2}\right]\right\rangle,
$$

where $\left\langle s^{-1} z ; s x\right\rangle=(-1)^{|z|}\langle z ; x\rangle$ and $\sharp L$ is the dual space of $L$.

Theorem 2.1 [24, §11],[10,25] For a Sullivan model $M(X)$ of $X$, Der $M(X)$ is a DGL-model of Baut $1 X$. In particular, there is an isomorphism of graded Lie algebras $H_{*}(\operatorname{Der} M(X)) \cong \pi_{*}\left(\Omega \mathrm{Baut}_{1} X\right)_{\mathbb{Q}}$ where the right hand has the Samelson bracket. Furthermore, $C^{*}(\operatorname{Der} M(X))$ is a DGA-model of $\operatorname{Baut}_{1} X$.

Two fibrations $\xi_{f_{1}}$ and $\xi_{f_{2}}$ are fibre homotopy equivalent if there is a diagram:

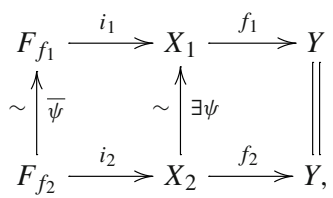

where $\psi \circ i_{2} \simeq i_{1} \circ \bar{\psi}$ and $f_{1} \circ \psi=f_{2}$. Then its Sullivan model is given as

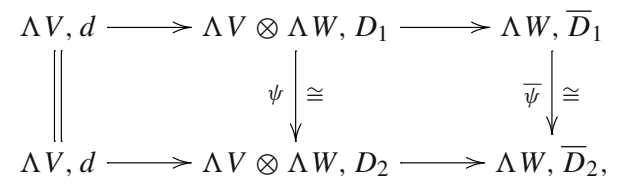

where the left square is DGA-commutative and the right square is DGA-homotopy commutative.

Lemma 2.2 Suppose that two maps $f_{1}$ and $f_{2}$ strictly induce $a_{f_{1}}$ and $a_{f_{2}}$, respectively. If $\xi_{f_{1}}$ and $\xi_{f_{2}}$ are fibre homotopy equivalent, there is a DGL-isomorphism $\phi: \operatorname{Der}\left(\Lambda V \otimes \Lambda W, D_{1}\right) \cong \operatorname{Der}\left(\Lambda V \otimes \Lambda W, D_{2}\right)$ such that $\phi(\sigma)=\psi \circ \sigma \circ \psi^{-1}$ and $b_{f_{2}} \circ \phi=b_{f_{1}}$. Thus, there is a homotopy equivalence map $\phi:\left(\text { Baut }_{1} X_{1}\right)_{0} \stackrel{\sim}{\rightarrow}$ (Baut $\left.X_{2}\right)_{0}$ such that $a_{f_{2}} \circ \phi=a_{f_{1}}$, i.e., $a_{f_{1}}$ and $a_{f_{2}}$ are fibre homotopy equivalent as fibrations. 
Proof Recall the DGA-diagram of $\S 1$. Then $D_{2}=\psi \circ D_{1} \circ \psi^{-1}$. Therefore, there is a DGL-isomorphism $\phi$ given by $\phi(\sigma)=\psi \circ \sigma \circ \psi^{-1}$ and

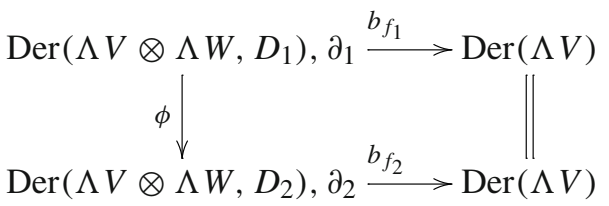

is DGL-commutative since $\left.\psi\right|_{\Lambda V}=i d_{\Lambda V}$. In particular, we can check $\partial_{2} \circ \phi=\phi \circ \partial_{1}$ by

$$
\begin{aligned}
\partial_{2} \phi(\sigma) & =D_{2} \psi \sigma \psi^{-1}-(-1)^{i}\left(\psi \sigma \psi^{-1}\right) D_{2}=\psi D_{1} \sigma \psi^{-1}-(-1)^{i} \psi \sigma D_{1} \psi^{-1} \\
& =\phi\left(D_{1} \sigma-(-1)^{i} \sigma D_{1}\right)=\phi \partial_{1}(\sigma)
\end{aligned}
$$

for $\sigma \in \operatorname{Der}_{i}\left(\Lambda V \otimes \Lambda W, D_{1}\right)$. Similarly, we have $\phi([\sigma, \tau])=[\phi(\sigma), \phi(\tau)]$.

Convention. For a DGA-model $(\Lambda V, d)$ the symbol $(v, f)$ means the elementary derivation that takes a generator $v$ of $V$ to an element $f$ of $\Lambda V$ and the other generators to 0 . Note that $|(v, f)|=|v|-|f|$.

Proof of Proposition 1.4 Let $M(Y)=(\Lambda V, d) \rightarrow(\Lambda V \otimes \Lambda W, D)$ be the model of $f$. Note that $b_{f}(\sigma)$ is a derivation on $\Lambda V$ for $\sigma \in \operatorname{Der}(\Lambda V \otimes \Lambda W)$ since

$$
\begin{aligned}
b_{f}(\sigma)(a \cdot b) & =\left(\operatorname{proj}_{V} \circ \sigma \circ i\right)(a \cdot b)=\operatorname{proj}_{V}\left(\sigma(a) \cdot b+(-1)^{|\sigma||a|} a \cdot \sigma(b)\right) \\
& =\left(\operatorname{proj}_{V} \circ \sigma \circ i\right)(a) \cdot b+(-1)^{|\sigma||a|} a \cdot\left(\operatorname{proj}_{V} \circ \sigma \circ i\right)(b)=b_{f}(\sigma)(a) \cdot b+(-1)^{|\sigma||a|} a \cdot b_{f}(\sigma)(b)
\end{aligned}
$$

for $a, b \in \Lambda V$. Thus, $b_{f}$ is well defined.

(if) When $\min W \geq \max V$, there is a decomposition of vector spaces

$$
\operatorname{Der}(\Lambda V \otimes \Lambda W)=\operatorname{Der}(\Lambda V) \oplus \operatorname{Der}(\Lambda W, \Lambda V \otimes \Lambda W)
$$

from degree arguments. Then there is a DGL-map $b_{f}: \operatorname{Der}(\Lambda V \otimes \Lambda W, D) \rightarrow \operatorname{Der}(\Lambda V, d)$ by $b_{f}\left(\sigma_{1}\right)=\sigma_{1}$ and $b_{f}\left(\sigma_{2}\right)=0$ for $\sigma=\sigma_{1}+\sigma_{2}$ with $\sigma_{1} \in \operatorname{Der}(\Lambda V)$ and $\sigma_{2} \in \operatorname{Der}(\Lambda W, \Lambda V \otimes \Lambda W)$. Then $b_{f}$ is a Lie algebra map since

$$
\begin{aligned}
b_{f}([\sigma, \tau]) & =b_{f}\left(\left[\sigma_{1}+\sigma_{2}, \tau_{1}+\tau_{2}\right]\right)=b_{f}\left(\left[\sigma_{1}, \tau_{1}\right]\right) \\
& =\operatorname{proj}_{V} \circ \sigma_{1} \circ \tau_{1} \circ i+(-1)^{\left|\sigma_{1} \| \tau_{1}\right|} \operatorname{proj}_{V} \circ \tau_{1} \circ \sigma_{1} \circ i \\
& =\left(\operatorname{proj}_{V} \circ \sigma_{1} \circ i\right) \circ\left(\operatorname{proj}_{V} \circ \tau_{1} \circ i\right)+(-1)^{\left|\sigma_{1}\right|\left|\tau_{1}\right|}\left(\operatorname{proj}_{V} \circ \tau_{1} \circ i\right) \circ\left(\operatorname{proj}_{V} \circ \sigma_{1} \circ i\right) \\
& =\left[b_{f}\left(\sigma_{1}\right), b_{f}\left(\tau_{1}\right)\right]=\left[b_{f}(\sigma), b_{f}(\tau)\right]
\end{aligned}
$$

for $\sigma, \tau \in \operatorname{Der}(\Lambda V \otimes \Lambda W)$. Furthermore, it preserves the differential since

$$
\begin{aligned}
\left(b_{f} \circ \partial_{X}\right)(\sigma) & =b_{f}\left(\partial_{X}\left(\sigma_{1}+\sigma_{2}\right)\right)=b_{f}\left(\partial_{X}\left(\sigma_{1}\right)\right)=b_{f}\left(\partial_{Y}\left(\sigma_{1}\right)+\tau\right) \\
& =b_{f}\left(\partial_{Y}\left(\sigma_{1}\right)\right)=\partial_{Y}\left(\sigma_{1}\right)=\partial_{Y}\left(b_{f}\left(\sigma_{1}\right)\right)=\partial_{Y}\left(b_{f}\left(\sigma_{1}+\sigma_{2}\right)\right)=\left(\partial_{Y} \circ b_{f}\right)(\sigma)
\end{aligned}
$$

for $\sigma \in \operatorname{Der}(\Lambda V \otimes \Lambda W)$ with some $\tau \in \operatorname{Der}(\Lambda W, \Lambda V \otimes \Lambda W)$.

(only if) Suppose that $\min W<\max V$. There are elements $w \in W$ and $v \in V$ with $|w|<|v|$. Then $b_{f}$ is not a DGL-map since $b_{f}([(w, 1),(v, w)])=b_{f}(v, 1) \neq 0$ but $\left[b_{f}(w, 1), b_{f}(v, w)\right]=[0,0]=0$ from the definition of $b_{f}$.

Example 2.3 Consider the case that $f$ is not $\pi_{\mathbb{Q}}$-separable (not $\mathbb{Q}$-w.t.). Let $f: S^{7} \rightarrow S^{4}$ be the Hopf map. Then the model is given by

$$
M\left(S^{4}\right)=(\Lambda(x, y), d) \rightarrow(\Lambda(x, y, z), D) \simeq M\left(S^{7}\right)
$$

with $|x|=4,|y|=7,|z|=3, d x=D x=0, D y=d y=x^{2}, D z=x, D y=x^{2}$. Then the bases of derivations are given as, 


\begin{tabular}{ll}
\hline$n$ & $\operatorname{Der}_{n}(\Lambda(x, y, z), D)$ \\
\hline 7 & $(y, 1)$ \\
4 & $(x, 1)(y, z)$ \\
3 & $(y, x)(z, 1)$ \\
1 & $(x, z)$ \\
\hline$n$ & $\operatorname{Der}_{n}(\Lambda(x, y), d)$ \\
\hline 7 & $(y, 1)$ \\
4 & $(x, 1)$ \\
3 & $(y, x)$ \\
\hline
\end{tabular}

where $H_{*}(\operatorname{Der}(\Lambda(x, y))=\mathbb{Q}\{(y, 1)\}$. By degree reason, any DGL-map

$$
\psi:(\operatorname{Der}(\Lambda(x, y, z), D) \rightarrow(\operatorname{Der}(\Lambda(x, y), d)
$$

is given by $\psi(y, 1)=a_{1}(y, 1), \psi(x, 1)=a_{2}(x, 1), \psi(y, z)=a_{3}(x, 1), \psi(y, x)=a_{4}(y, x), \psi(z, 1)=$ $a_{5}(y, x)$ and $\psi_{f}(x, z)=0$ for some $a_{i} \in \mathbb{Q}$.

From $(x, 1)=[(z, 1),(x, z)]$ and $(y, z)=[(x, z),(y, x)]$ we have $a_{2}=0$ and $a_{3}=0$, respectively. Then from $2(y, 1)=[(z, 1),(y, z)]+[(x, 1),(y, x)]$, we obtain $a_{1}=0$. Thus, $\|\psi\|$ is homotopic to the constant map.

Let $f: X \rightarrow Y$ be a map with a section $s$, i.e., there is a map $s: Y \rightarrow X$ with $f \circ s \sim i d_{Y}$. Then there is a map $\psi_{f}:$ aut $_{1} X \rightarrow \operatorname{aut}_{1} Y$ with $\psi_{f}(g):=f \circ g \circ s$ for $g \in \operatorname{aut}_{1} X$. In general, this does not preserve the monoid structures.

Theorem 2.4 If a $\pi_{\mathbb{Q}}$-separable map $f$ admits a section, there is a commutative diagram:

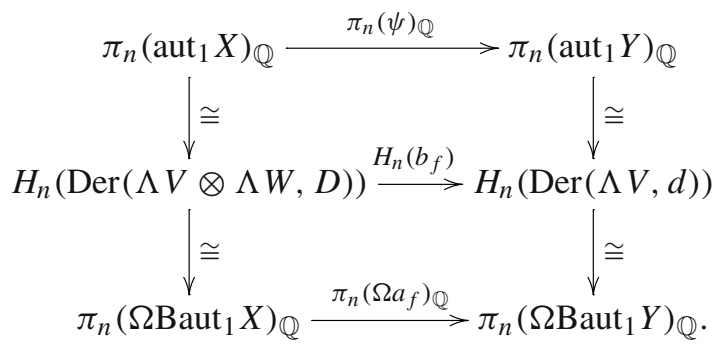

Proof The map $\pi_{n}\left(\psi_{f}\right): \pi_{n}\left(\operatorname{aut}_{1} X\right) \rightarrow \pi_{n}\left(\right.$ aut $\left._{1} Y\right)$ is given by $\pi_{n}\left(\psi_{f}\right)([\sigma])=[\tau]:=\left[f \circ \sigma \circ\left(s \times 1_{S^{n}}\right)\right]$ in the following homotopy commutative diagram:

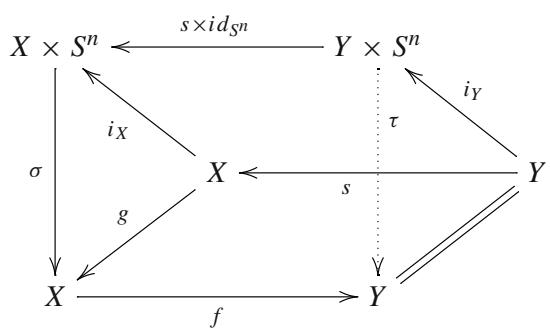

from adjointness. That is the pointed homotopy classes of maps $S^{n} \rightarrow \operatorname{aut}_{1} X=\operatorname{map}\left(X, X ; i d_{X}\right)$ are in bijection with the homotopy classes of those maps $X \times S^{n} \rightarrow X$ that composed with the inclusion $i_{X}: X \rightarrow X \times S^{n}$ yield the identity [20, p. 43-44]. Let $M(Y)=(\Lambda V, d) \stackrel{i}{\rightarrow}(\Lambda V \otimes \Lambda W, D) \simeq M(X)$ be the model of $f$. We identify the rationalized map $\sigma_{0}:\left(X \times S^{n}\right)_{0} \rightarrow X_{0}$ to an element of $\operatorname{Der}_{n}(\Lambda V \otimes \Lambda W)$ [24, p. 313] (cf. [20, Proposition 11]). Then there is a chain map

$$
c_{f}: \operatorname{Der}(\Lambda V \otimes \Lambda W, D) \rightarrow \operatorname{Der}(\Lambda V, d)
$$


given by $c_{f}\left(\sigma_{0}\right)=\operatorname{proj}_{V} \circ \sigma_{0} \circ i$. It is well defined, i.e., $\partial_{Y} \circ c_{f}=c_{f} \circ \partial_{X}$, from $D W \subset \Lambda V \otimes \Lambda^{+} W$ [26] since it admits a section. (However, $c_{f}$ is not a DGL-map in general.) Notice that

$$
\pi_{n}\left(\psi_{f}\right)_{\mathbb{Q}}\left(\left[\sigma_{0}\right]\right) \equiv H_{n}\left(c_{f}\right)\left(\left[\sigma_{0}\right]\right)
$$

If $f$ is a $\pi_{\mathbb{Q}}$-separable map, $c_{f}$ is the DGL-map $b_{f}$.

The following is obvious from the definition of $b_{f}$ and useful:

Claim 2.5 For any $\pi_{\mathbb{Q}}$-separable map $f: X \rightarrow Y$, we have $b_{f}(C)=0$ and $\left.b_{f}\right|_{\operatorname{Der}(\Lambda V)}=i d_{\operatorname{Der}(\Lambda V)}$ for $\operatorname{Der}(\Lambda V \otimes \Lambda W)=C \oplus \operatorname{Der}(\Lambda V)$.

Theorem 2.6 For a $\pi_{\mathbb{Q}}$-separable map $f: X \rightarrow Y$, let

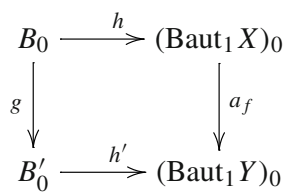

be a commutative diagram. Then there exists a map between total spaces $k: E \rightarrow E^{\prime}$ in the diagram:

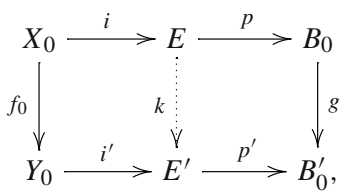

where $k \circ i \simeq i^{\prime} \circ f_{0}$ and $g \circ p=p^{\prime} \circ k$. Here $p: E \rightarrow B_{0}$ and $p^{\prime}: E^{\prime} \rightarrow B_{0}^{\prime}$ are induced by the rationalized classifying maps $h$ and $h^{\prime}$, respectively.

Proof Let $X \rightarrow E_{\infty}^{X} \stackrel{p_{\infty}^{X}}{\rightarrow}$ Baut $_{1} X$ and $Y \rightarrow E_{\infty}^{Y} \stackrel{p_{\infty}^{Y}}{\rightarrow}$ Baut $_{1} Y$ be the universal fibrations of $X$ and $Y$, respectively. Let $C^{*}(\operatorname{Der}(\Lambda V)) \otimes \Lambda V, D_{Y}$ be the DGA-model of $E_{\infty}^{Y}$ and $C^{*}(\operatorname{Der}(\Lambda V \otimes \Lambda W)) \otimes \Lambda V \otimes \Lambda W, D_{X}$ be the DGA-model of $E_{\infty}^{X}$. For a $\pi_{\mathbb{Q}}$-separable map $f: X \rightarrow Y$, there exists a DGA-inclusion map $\psi$ such that

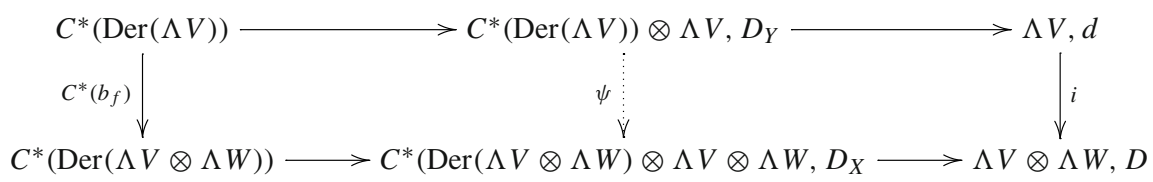

is commutative from the universality. Indeed, $C^{*}(\operatorname{Der}(\Lambda V)) \otimes \Lambda V, D_{Y}$ is a sub-DGA of $C^{*}(\operatorname{Der}(\Lambda V \otimes \Lambda W)) \otimes$ $\Lambda V \otimes \Lambda W, D_{X}$ from Claim 2.5 and $\max V \leq \min W$. Thus, there is a map $\tilde{a}_{f}:=|\psi|:\left(E_{\infty}^{X}\right)_{0} \rightarrow\left(E_{\infty}^{Y}\right)_{0}$ such that $\left(p_{\infty}^{Y}\right)_{0} \circ \tilde{a}_{f}=a_{f} \circ\left(p_{\infty}^{X}\right)_{0}$. Since $p^{\prime}$ is the pull-back of $\left(p_{\infty}^{Y}\right)_{0}$ by $h^{\prime}$, there exists a map $k: E \rightarrow E^{\prime}$ such that

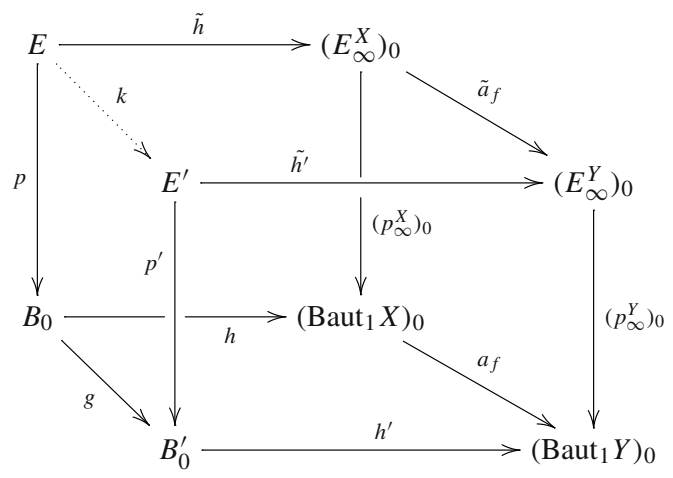

is commutative from the universality since $h^{\prime} \circ g \circ p=a_{f} \circ h \circ p=a_{f} \circ\left(p_{\infty}^{X}\right)_{0} \circ \tilde{h}=\left(p_{\infty}^{Y}\right)_{0} \circ \tilde{a}_{f} \circ \tilde{h}$. 
Example 2.7 Let $X=K(\mathbb{Q}, n) \times K(\mathbb{Q}, 2 n)$ and $Y=K(\mathbb{Q}, n)$ for some even integer $n$. Then $M(X)=$ $\Lambda(x, y), 0$ and $M(Y)=\Lambda(z), 0$ with $|x|=|z|=n$ and $|y|=2 n$. Let a map $f: X \rightarrow Y$ be given by $M(f): \Lambda(z) \rightarrow \Lambda(x, y)$ with $M(f)(z)=x$. The homotopy fibration of any $\pi_{\mathbb{Q}}$-separable map is given by $\Lambda(z), 0 \rightarrow \Lambda(z, y), 0 \cong \Lambda(x, y), 0$ from the degree reason. Therefore, the DGL-map $\psi: \operatorname{Der} \Lambda(x, y) \rightarrow$ $\operatorname{Der} \Lambda(z)$ such that $\psi((y, x))=\psi((x, 1))=(z, 1)$ is not DGL-homotopic to $b_{f}$ from Claim 2.5.

Let $h: S_{0}^{n+1} \rightarrow\left(\text { Baut }_{1} X\right)_{0}$ and $h^{\prime}: S_{0}^{n+1} \rightarrow\left(\operatorname{Baut}_{1} Y\right)_{0}$ be given by $L(h): \mathbb{L}(u) \rightarrow \operatorname{Der}(\Lambda(x, y))$ with $|u|=n, L(h)(u)=(y, x)$ and $L\left(h^{\prime}\right): \mathbb{L}(u) \rightarrow \operatorname{Der}(\Lambda(z))$ with $L\left(h^{\prime}\right)(u)=(z, 1)$, respectively. Then the commutative diagram

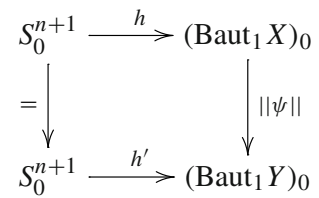

does not induce a map between total spaces $f^{\prime}: E \rightarrow E^{\prime}$ such that

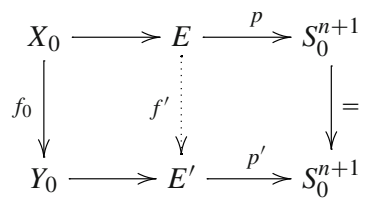

is homotopy commutative. Indeed, there does not exist a DGA-map $h: \Lambda(v, z), D^{\prime} \rightarrow \Lambda(v, x, y), D$ with $D^{\prime} z=v, D y=v x$ and $D x=0$ such that

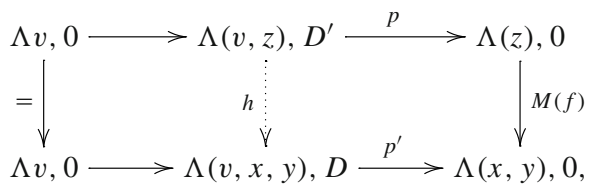

where $|v|=n+1$ is homotopy commutative since $h$ cannot be a DGA-map from $D h(z)=0$ but $h D^{\prime}(z)=v$.

\section{When does $a_{f}$ admit a section?}

Let $f: X \rightarrow Y$ be a $\pi_{\mathbb{Q}}$-separable map with homotopy fiber $F_{f}$ and $\operatorname{Der}(\Lambda W, \Lambda V \otimes \Lambda W)$ the sub-DGL of $\operatorname{Der}(\Lambda V \otimes \Lambda W)$ restricted to derivations out of $\Lambda W$.

Proposition 3.1 Let $F_{a_{f}}$ be the homotopy fiber of $a_{f}$. Then the DGL-model of the fibration $\chi_{f}: F_{a_{f}} \stackrel{j}{\rightarrow}$ $\left(\text { Baut }_{1} X\right)_{0} \stackrel{a_{f}}{\rightarrow}\left(\text { Baut }_{1} Y\right)_{0}$ is given by

$$
\operatorname{Der}(\Lambda W, \Lambda V \otimes \Lambda W) \stackrel{\text { incl. }}{\rightarrow} \operatorname{Der}(\Lambda V \otimes \Lambda W) \stackrel{b_{f}}{\rightarrow} \operatorname{Der}(\Lambda V) .
$$

Proof Since $b_{f}$ is surjective and $\operatorname{Der}(\Lambda W, \Lambda V \otimes \Lambda W)$ is $\operatorname{Ker} b_{f}$, it follows from [25, VI.1.(3) Proposition].

Let $L(F)=\oplus_{i>0} L(F)_{i}$ be the degree decomposition of a DGL-model of a space $F$.

Theorem 3.2 $L\left(F_{a_{f}}\right)_{n} \cong \oplus_{i-j=n} \operatorname{Der}_{i}(\Lambda W) \otimes H^{j}(\Lambda V)$.

Proof A chain-map $\rho: \operatorname{Der}_{i}(\Lambda W) \otimes H^{j}(\Lambda V) \rightarrow \operatorname{Der}_{i}\left(\Lambda W, \Lambda W \otimes(\Lambda V)^{j}\right)$ is given by $\rho(\sigma \otimes$ $[f])(w):=(-1)^{|w| j} \sigma(w) \cdot f$ induced by an inclusion $H^{j}(\Lambda V) \rightarrow(\Lambda V)^{j}$. It is quasi-isomorphic, i.e., there is a decomposition $\operatorname{Der}(\Lambda W, \Lambda W \otimes \Lambda V)=\left(\operatorname{Der}(\Lambda W) \otimes H^{*}(\Lambda V)\right) \oplus C$ for a complex $C$ of derivations with $H_{*}(C)=0$. 
The rational homotopy exact sequence of the strictly induced fibration $\chi_{f}$ :

$$
\begin{gathered}
\ldots \stackrel{j_{\sharp}}{\rightarrow} \pi_{n+2}\left(\text { Baut }_{1} X\right)_{\mathbb{Q}} \stackrel{a_{f_{\sharp}}}{\rightarrow} \pi_{n+2}\left(\text { Baut }_{1} Y\right)_{\mathbb{Q}} \stackrel{\delta_{f}}{\rightarrow} \\
\pi_{n+1}\left(F_{a_{f}}\right)_{\mathbb{Q}} \stackrel{j_{\sharp}}{\rightarrow} \pi_{n+1}\left(\text { Baut }_{1} X\right)_{\mathbb{Q}} \stackrel{a_{f_{\sharp}}}{\rightarrow} \pi_{n+1}\left(\text { Baut }_{1} Y\right)_{\mathbb{Q}} \stackrel{\delta_{f}}{\rightarrow} \ldots
\end{gathered}
$$

is equivalent to the homology exact sequence:

$$
\begin{gathered}
\cdots \rightarrow H_{n+1}(\operatorname{Der}(\Lambda V \otimes \Lambda W)) \stackrel{b_{f_{*}}}{\rightarrow} H_{n+1}(\operatorname{Der}(\Lambda V)) \stackrel{\delta_{f}}{\rightarrow} \\
H_{n}(\operatorname{Der}(\Lambda W, \Lambda V \otimes \Lambda W)) \rightarrow H_{n}(\operatorname{Der}(\Lambda V \otimes \Lambda W)) \stackrel{b_{f_{*}}}{\rightarrow} H_{n}(\operatorname{Der}(\Lambda V)) \stackrel{\delta_{f}}{\rightarrow} \cdots
\end{gathered}
$$

Then we have the following from an ordinary chain complex property:

Claim 3.3 The connecting map $\delta_{f}$ is given by $\delta_{f}([\sigma])=[\tau]$ when $\partial_{X}(\sigma)=\tau$ for a $\partial_{Y}$-cycle $\sigma$ of $\operatorname{Der}(\Lambda V)$ and $a \partial_{X}$-cycle $\tau$ of $\operatorname{Der}(\Lambda W, \Lambda V \otimes \Lambda W)$.

Recall that the following implications hold for a general fibration $\chi: F \rightarrow E \stackrel{p}{\rightarrow} B$ of simply connected spaces:

$$
\chi \text { is fibre-trivial } \Rightarrow p \text { admits a section } \Rightarrow \chi \text { is weakly trivial } \Leftrightarrow \delta=0 \text {. }
$$

Here $\delta: \pi_{*}(B) \rightarrow \pi_{*-1}(F)$ is the connecting map of the homotopy exact sequence for $\chi$. The following may be a characteristic phenomenon in our fibration $\chi_{f}$.

Proposition $3.4 a_{f}$ admits a section if and only if $\delta_{f}=0$.

Proof (if) Let the DGA-model of the fibration $\chi_{f}: F_{a_{f}} \stackrel{j}{\rightarrow}\left(\operatorname{Baut}_{1} X\right)_{0} \stackrel{a_{f}}{\rightarrow}\left(\operatorname{Baut}_{1} Y\right)_{0}$ be given as the commutative diagram:

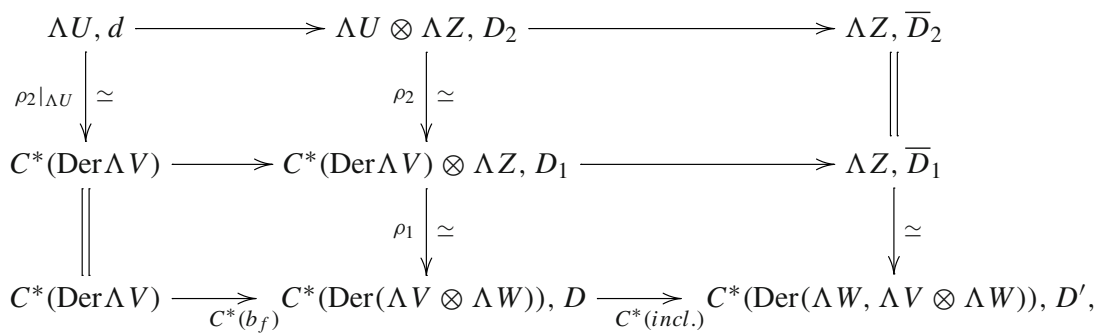

where $M\left(\operatorname{Baut}_{1} Y\right) \cong(\Lambda U, d)$ with $U^{n+1}=H_{n}(\operatorname{Der}(\Lambda V))$ and $M\left(F_{a_{f}}\right) \cong\left(\Lambda Z, \bar{D}_{2}\right)$ with $Z^{n+1}=$ $H_{n}(\operatorname{Der}(\Lambda W, \Lambda V \otimes \Lambda W))$. Here

$$
(\Lambda U, d) \rightarrow\left(\Lambda U \otimes \Lambda Z, D_{2}\right) \rightarrow\left(\Lambda Z, \bar{D}_{2}\right)
$$

is the model of $\chi_{f}$. From the assumption $\chi_{f}$ is weakly equivalent, i.e., $M\left(\right.$ Baut $\left._{1} X\right) \cong\left(\Lambda U \otimes \Lambda Z, D_{2}\right)$ as a minimal model. Let $D=d_{1}+d_{2}$ as in $\$ 2$. Notice that a linear component of any bracket representation of $\sigma \in \operatorname{Der}(\Lambda W, \Lambda V \otimes \Lambda W)$ is not contained in $[\operatorname{Der}(\Lambda V)$, $\operatorname{Der}(\Lambda V)]$, where [, ] is the Lie bracket. That means

$$
d_{2}\left(s^{-1} \sigma^{*}\right) \in I\left(C^{*}(\operatorname{Der}(\Lambda W, \Lambda V \otimes \Lambda W))\right) \quad(* *) .
$$

Here $I(S)$ is the ideal in $C^{*}(\operatorname{Der}(\Lambda V \otimes \Lambda W))$ generated by a basis of a vector space $S$. Let $\sigma$ be a non-exact $\partial_{X}$-cycle of $\operatorname{Der}(\Lambda W, \Lambda V \otimes \Lambda W)$. Then

$$
H^{*}\left(C^{*}(\operatorname{Der}(\Lambda W, \Lambda V \otimes \Lambda W)), d_{1}^{\prime}\right) / H^{*} \cdot H^{*} \cong Z \ni\left[s^{-1} \sigma^{*}\right]
$$

for $D^{\prime}=d_{1}^{\prime}+d_{2}^{\prime}$ as in $\S 2$. Since $\rho_{1}\left(D_{1}\left(\left[s^{-1} \sigma^{*}\right]\right)\right)$ and $d_{2}\left(s^{-1} \sigma^{*}\right)$ are $D$-cohomologous in $C^{*}(\operatorname{Der}(\Lambda V \otimes \Lambda W))$, we can put

$$
D_{1}\left(\left[s^{-1} \sigma^{*}\right]\right) \in I(Z)
$$

from (**), i.e., $D_{1}(Z) \subset C^{*}(\operatorname{Der} \Lambda V) \otimes \Lambda^{+} Z$. By $\rho_{2}, D_{2}(Z) \subset \Lambda U \otimes \Lambda^{+} Z$. Then we have done from [26]. (only if) It holds from the above implications $(*)$. 
Theorem 3.5 If a $\pi_{\mathbb{Q}}$-separable map $f: X \rightarrow Y$ is rationally fibre-trivial (i.e., $\left.X_{0} \sim\left(F_{f}\right)_{0} \times Y_{0}\right)$, a $a_{f}$ admits a section.

Proof From the assumption and Claim 3.3, we have $\delta_{f}=0$. Then it holds from Proposition 3.4.

Refer [19, page 292] for related topics. Conversely, when $Y$ is an odd-sphere,

Theorem 3.6 If a $\pi_{\mathbb{Q}}$-separable map $f: X \rightarrow Y=S^{2 n+1}$ is not rationally fibre-trivial, $a_{f}$ does not admit a section. Furthermore, $a_{f} \sim *($ the constant map).

Proof Let $M\left(S^{2 n+1}\right)=(\Lambda v, 0)$. Since there exists an element $w \in W$ such that $D w \in \Lambda v \otimes \Lambda^{+} W$ from the assumption, $\partial_{X}(v, 1)= \pm(w, \partial D w / \partial v)+\cdots \neq 0$ in $\operatorname{Der}(\Lambda W)$. From Claim $3.3 \delta_{f}$ is injective since $\delta_{f}([(v, 1)])=[ \pm(w, \partial D w / \partial v)+\cdots] \neq 0$. Then the former holds from Proposition 3.4. Furthermore, from the homotopy exact sequence, we have $a_{f_{\sharp}}=0$. Thus, the latter holds.

Example 3.7 (1) Let $S^{5} \times S^{7} \rightarrow X \rightarrow Y=S^{3}$ be a non-(fibre-)trivial $\pi_{\mathbb{Q}}$-separable fibration given by the model

$$
\left(\Lambda\left(v_{1}\right), 0\right) \rightarrow\left(\Lambda\left(v_{1}, w_{1}, w_{2}\right), D\right) \rightarrow\left(\Lambda\left(w_{1}, w_{2}\right), 0\right)
$$

with $\left|v_{1}\right|=3,\left|w_{1}\right|=5,\left|w_{2}\right|=7, D w_{1}=0$ and $D w_{2}=v_{1} w_{1}$. Then $a_{f}$ does not admit a section from Theorem 3.6. Indeed $\delta_{f}: H_{3}\left(\operatorname{Der}\left(\Lambda v^{\prime}\right)\right) \rightarrow H_{2}\left(\operatorname{Der}\left(\Lambda\left(w_{1}, w_{2}\right), \Lambda\left(v_{1}, w_{1}, w_{2}\right)\right)\right)$ is non-trivial from $\delta_{f}\left(\left[\left(v_{1}, 1\right)\right]\right)=\left[\left(w_{2}, w_{1}\right)\right] \neq 0$.

(2) Let $S^{5} \times S^{7} \rightarrow X^{\prime} \rightarrow Y^{\prime}$ be a non-(fibre-)trivial $\pi_{\mathbb{Q}^{-s e p a r a b l e ~ f i b r a t i o n ~ g i v e n ~ b y ~ t h e ~ m o d e l ~}}$

$$
\left(\Lambda\left(v_{1}, v_{2}, v_{3}\right), d_{Y^{\prime}}\right) \rightarrow\left(\Lambda\left(v_{1}, v_{2}, v_{3}, w_{1}, w_{2}\right), D^{\prime}\right) \rightarrow\left(\Lambda\left(w_{1}, w_{2}\right), 0\right)
$$

with $\left|v_{1}\right|=\left|v_{2}\right|=3,\left|v_{3}\right|=5,\left|w_{1}\right|=7,\left|w_{2}\right|=9, d_{Y^{\prime}}\left(v_{1}\right)=d_{Y^{\prime}}\left(v_{2}\right)=0, d_{Y^{\prime}}\left(v_{3}\right)=v_{1} v_{2}$, $D^{\prime} w_{1}=0$ and $D^{\prime} w_{2}=v_{1} w_{1}$. Then $a_{f}$ admits a section from Proposition 3.4 since $\delta_{f}\left(\left[\left(v_{3}, 1\right)\right]\right)=0$ for $H_{*}\left(\operatorname{Der}\left(\Lambda\left(v_{1}, v_{2}, v_{3}\right)\right)\right)=\mathbb{Q}\left\{\left[\left(v_{3}, 1\right)\right]\right\}$. However, $\chi_{f}$ is not trivial from $\left[\left(v_{3}, 1\right),\left(w_{2}, v_{2} v_{3}\right)\right]=\left(w_{2}, v_{2}\right)$. Indeed, then

$$
\mathcal{D}\left(s^{-1}\left(w_{2}, v_{2}\right)^{*}\right)=d_{2}\left(s^{-1}\left(w_{2}, v_{2}\right)^{*}\right)=s^{-1}\left(v_{3}, 1\right)^{*} \cdot s^{-1}\left(w_{2}, v_{2} v_{3}\right)^{*}
$$

for $\left(C^{*}\left(\operatorname{Der}\left(\Lambda\left(v_{1}, v_{2}, v_{3}, w_{1}, w_{2}\right)\right), \mathcal{D}\right)\right.$ with $\mathcal{D}=d_{1}+d_{2}$. Refer the proof of Proposition 3.4.

Proof of Theorem 1.7 Let $M(Y)=(\Lambda V, d)=\left(\mathbb{Q}\left[x_{1}, \ldots, x_{n}\right] \otimes \Lambda\left(y_{1}, \ldots, y_{n}\right), d\right)$ with $d x_{i}=0$ and $d y_{i}=f_{i}$ for $i=1, . ., n$.

(if) Let $M(Y)=(\Lambda V, d) \rightarrow(\Lambda V \otimes \Lambda W, D)$ be the model of $f$. From the regularity of $f_{1}, \ldots, f_{n}$, $\operatorname{Im} D \subset \mathbb{Q}\left[x_{1}, \ldots, x_{n}\right] \otimes \Lambda W$. Thus,

$$
\partial_{X}\left(x_{i}, h_{i}\right)=\sum_{j=1}^{n}\left(y_{j},\left(\partial f_{j} / \partial x_{i}\right) \cdot h_{i}\right)+\theta \text { and } \partial_{X}\left(y_{i}, h_{i}\right)=0 \quad(i=1, \ldots, n)
$$

for any $h_{i} \in \Lambda V^{\text {even }}=\mathbb{Q}\left[x_{1}, \ldots, x_{n}\right]$ with suitable degree and some $\theta \in \operatorname{Der}(\Lambda W, \Lambda V \otimes \Lambda W)$. Then we have $\delta_{f}=0$ from Claim 3.3 since $H_{\text {even }}(\operatorname{Der} M(Y))=0$ [18] from the assumption. Then $a_{f}$ admits a section from Proposition 3.4. Furthermore, from Theorem 3.2, we can suppose that the Lie bracket decomposition of an element of $\operatorname{Der}(\Lambda W, \Lambda V \otimes \Lambda W)$ does not have an element of $\operatorname{Der}(\Lambda V)$ as a factor since $\operatorname{Der} H^{*}(Y ; \mathbb{Q})=0$ [18] again. Thus, we have

$$
D_{2}=d \otimes 1 \pm 1 \otimes \bar{D}_{2}
$$

for the Sullivan minimal model $(\Lambda U, d) \rightarrow\left(\Lambda U \otimes \Lambda Z, D_{2}\right) \rightarrow\left(\Lambda Z, \bar{D}_{2}\right)$ of $\chi_{f}$ (in the proof of Proposition 3.4).

(only if) Suppose that $Y$ does not satisfies the Halperin conjecture, i.e., there is a non-zero element $\left[\sum_{i}\left(x_{i}, h_{i}\right)+\sum_{j}\left(y_{j}, g_{j}\right)\right] \in H_{2 m}(\operatorname{Der} M(Y))$ for $h_{i} \in \mathbb{Q}\left[x_{1}, \ldots, x_{n}\right], g_{j} \in \Lambda V$ and some $m$ [18]. Let $\left(S^{a} \times S^{b} \simeq_{0}\right) F \rightarrow X \stackrel{f}{\rightarrow} Y$ be a fibration of the model:

$$
(\Lambda V, d) \rightarrow\left(\Lambda V \otimes \Lambda\left(w_{1}, w_{2}, w_{3}\right), D\right) \rightarrow\left(\Lambda\left(w_{1}, w_{2}, w_{3}\right), d_{F}\right),
$$


where $\left|w_{1}\right|=a$ is even and $\left|w_{2}\right|=b$ is odd with $b-a=\left|x_{k}\right|-1$ for some $k, d_{F} w_{1}=d_{F} w_{2}=0, d_{F} w_{3}=w_{1}^{2}$, $D w_{1}=0$ and $D w_{2}=x_{k} w_{1}$. When $h_{k}$ is not $d_{Y}$-exact, the element $h_{k} w_{1}$ is not $D$-exact. Then

$$
\delta_{f}\left(\left[\sum_{i}\left(x_{i}, h_{i}\right)+\sum_{j}\left(y_{j}, g_{j}\right)\right]\right)=\left[\left(w_{2}, h_{k} w_{1}\right)\right] \neq 0
$$

for $\delta_{f}: H_{2 m}(\operatorname{Der} \Lambda V) \rightarrow H_{2 m-1}\left(\operatorname{Der}\left(\Lambda\left(w_{1}, w_{2}, w_{3}\right), \Lambda V \otimes \Lambda\left(w_{1}, w_{2}, w_{3}\right)\right)\right.$ from Claim 3.3. In particular, $\chi_{f}$ is not fibre-trivial.

Example 3.8 Let $Y$ be the homogeneous space $S U(6) / S U(3) \times S U(3)$. Then $Y$ is a pure space but not an $F_{0}$-space since rank $S U(6)=5>4=\operatorname{rank}(S U(3) \times S U(3))$. Let $\xi:\left(S^{11} \times S^{23} \simeq_{0}\right) F \rightarrow X \stackrel{f}{\rightarrow} Y$ be a fibration whose relative model is given as

$$
\left(\Lambda\left(x_{1}, x_{2}, y_{1}, y_{2}, y_{3}\right), d_{Y}\right) \rightarrow\left(\Lambda\left(x_{1}, x_{2}, y_{1}, y_{2}, y_{3}\right) \otimes \Lambda\left(w_{1}, w_{2}\right), D\right) \rightarrow\left(\Lambda\left(w_{1}, w_{2}\right), 0\right),
$$

where $\left|x_{1}\right|=4,\left|x_{2}\right|=6,\left|y_{1}\right|=7,\left|y_{2}\right|=9,\left|y_{3}\right|=11,\left|w_{1}\right|=11,\left|w_{2}\right|=23, d_{Y} y_{1}=x_{1}^{2}$, $d_{Y} y_{2}=x_{1} x_{2}, d_{Y} y_{3}=x_{2}^{2}, D w_{1}=0$ and $D w_{2}=\left(x_{1} y_{2}-x_{2} y_{1}\right) w_{1}$. Then $\partial_{X}\left(\left(y_{1}, 1\right)\right)=\left(w_{2}, x_{2} w_{1}\right)$, i.e., $\delta_{f}\left[\left(y_{1}, 1\right)\right]=\left[\left(w_{2}, x_{2} w_{1}\right)\right] \neq 0$ from Claim 3.3. In particular, $\chi_{f}$ is not trivial. Refer [19, Example 1.14(2)] for the Sullivan minimal model of $\operatorname{Baut}_{1} Y$.

\section{The obstruction class for a lifting}

Let $L(B)=\left(L(B), \partial_{B}\right)$ be the Quillen model of a simply connected CW complex $B$ of finite type. Then $L\left(B \cup_{\alpha} e^{N}\right)$ is given by $L(B) \bigsqcup \mathbb{L}(u), \partial_{\alpha}$ where $|u|=N-1,\left.\partial_{\alpha}\right|_{L(B)}=\partial_{B}$ and $\partial_{\alpha}(u) \in L(B)$ [25, Proposition III.3.(6)].

Theorem 4.1 For a $\pi_{\mathbb{Q}}$-separable map $f: X \rightarrow Y$, let

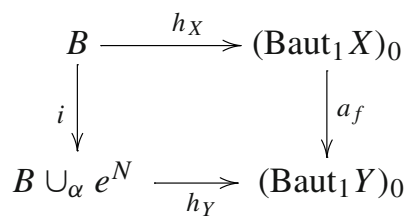

be a commutative diagram. Then there is a lift $h$ such that

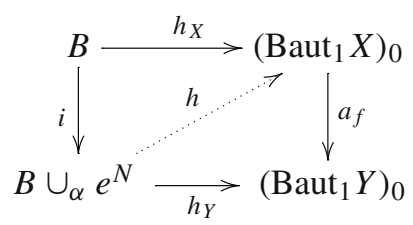

is commutative if and only if

$$
\mathcal{O}_{\alpha}\left(h_{X}, h_{Y}\right):=\left[\tau\left(h_{Y}(u)\right)-h_{X}^{\prime \prime}\left(\partial_{\alpha}(u)\right)\right]=0
$$

in $H_{N-2}(\operatorname{Der}(\Lambda W, \Lambda V \otimes \Lambda W))=\pi_{N-1}\left(F_{a_{f}}\right)_{\mathbb{Q}}$ for the DGL-commutative diagram

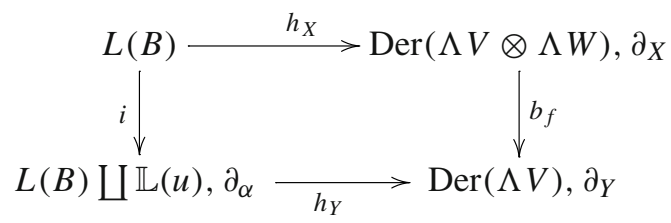

with 
- $\partial_{X} \mid \operatorname{Der}(\Lambda V, \Lambda V \otimes \Lambda W)=\partial_{Y}+\tau$ and $\partial_{X} \mid \operatorname{Der}(\Lambda W, \Lambda V \otimes \Lambda W)=\tau$ for some $\tau: \operatorname{Der}_{*}(\Lambda V \otimes \Lambda W) \rightarrow$ $\operatorname{Der}_{*-1}(\Lambda W, \Lambda V \otimes \Lambda W)$ and

- $h_{X}=h_{X}^{\prime}+h_{X}^{\prime \prime}$ where $h_{X}^{\prime}(b) \in \operatorname{Der}(\Lambda V)$ and $h_{X}^{\prime \prime}(b) \in \operatorname{Der}(\Lambda W, \Lambda V \otimes \Lambda W)$ for $b \in L(B)$.

Proof Since $b_{f} \circ h_{X}=h_{Y} \circ i$ and $h_{Y}$ is a DGL-map,

$$
h_{X}^{\prime} \partial_{\alpha}(u)=h_{Y} \partial_{\alpha}(u)=\partial_{Y} h_{Y}(u)
$$

in $\operatorname{Der}(\Lambda V)$. Notice that the obstruction element $\partial_{X}\left(h_{Y}(u)\right)-h_{X}\left(\partial_{\alpha}(u)\right)$ is a $\partial_{X}$-cycle in $\operatorname{Der}(\Lambda V \otimes \Lambda W)$. Therefore, $\tau\left(h_{Y}(u)\right)-h_{X}^{\prime \prime}\left(\partial_{\alpha}(u)\right)$ is a $\partial_{X}$-cycle in $\operatorname{Der}(\Lambda W, \Lambda V \otimes \Lambda W)$ from (1).

(if) Suppose that $\mathcal{O}_{\alpha}\left(h_{X}, h_{Y}\right)=0$. Then there is an element $q \in \operatorname{Der}(\Lambda W, \Lambda V \otimes \Lambda W)$ such that

$$
\partial_{X}(q)=\tau\left(h_{Y}(u)\right)-h_{X}^{\prime \prime}\left(\partial_{\alpha}(u)\right)
$$

Let

$$
\left.h\right|_{L(B)}:=h_{X} \text { and } h(u):=h_{Y}(u)-q .
$$

Then $h$ is a DGL-map since

$$
\begin{aligned}
\partial_{X}(h(u)) & =\partial_{X}\left(h_{Y}(u)\right)-\partial_{X}(q) \\
& =\left(h_{X}^{\prime} \partial_{\alpha}(u)+\tau h_{Y}(u)\right)-\left(\tau h_{Y}(u)-h_{X}^{\prime \prime} \partial_{\alpha}(u)\right) \\
& =h_{X}^{\prime} \partial_{\alpha}(u)+h_{X}^{\prime \prime} \partial_{\alpha}(u)=h_{X}\left(\partial_{\alpha}(u)\right)=h\left(\partial_{\alpha}(u)\right)
\end{aligned}
$$

from (1) and (2). Furthermore,

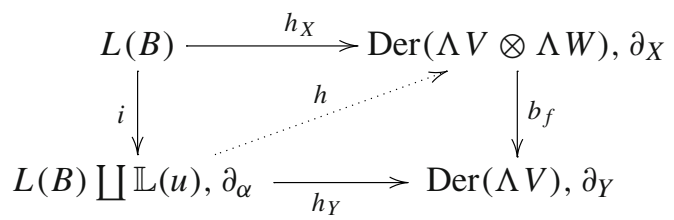

is commutative since $b_{f}(q)=0$. Thus, the (if)-part holds from the special realization of $(*)$.

(only if) Suppose that there exists a map $h$ such that $(*)$ is commutative. Since $h$ is a DGL-map,

$$
\partial_{X}(h(u))=h\left(\partial_{\alpha}(u)\right)
$$

in $\operatorname{Der}(\Lambda V \otimes \Lambda W)$ and

$$
h_{X}^{\prime \prime} \partial_{\alpha}(u)=\tau h(u)
$$

from (1) and (3). Furthermore,

$$
\tau h(u) \sim \tau h_{Y}(u)
$$

in $\operatorname{Der}(\Lambda W, \Lambda V \otimes \Lambda W)$. Here $\sim$ means "homologous". Indeed, (5) follows since

$$
h(u)=h_{Y}(u)+x
$$

for some element $x \in \operatorname{Der}(\Lambda W, \Lambda V \otimes \Lambda W)$ from $b_{f} \circ h=h_{Y}$ and then since

$$
\tau h(u)=\tau\left(h_{Y}(u)+x\right)=\tau h_{Y}(u)+\partial_{X}(x) .
$$

Thus, we obtain that $\mathcal{O}_{\alpha}\left(h_{X}, h_{Y}\right)=\left[\tau\left(h_{Y}(u)\right)-h_{X}^{\prime \prime}\left(\partial_{\alpha}(u)\right)\right]=0$ from (4) and (5).

From Theorem 3.2, we have

Corollary 4.2 If $\pi_{\geq N-1}\left(\text { Baut }_{1} F_{f}\right)_{\mathbb{Q}}=0$ for the homotopy fiber $F_{f}$ of $f$, there exists a lift $h$ for the pair $\left(h_{X}, h_{Y}\right)$ of above. 
Example 4.3 Let $B=S^{2}=\mathbb{C} P^{1}$. Let $S^{3} \times S^{5} \rightarrow X \stackrel{f}{\rightarrow} Y=S^{3}$ be the fibration given by the model

$$
(\Lambda(v), 0) \rightarrow\left(\Lambda\left(v, w_{1}, w_{2}\right), D\right) \rightarrow\left(\Lambda\left(w_{1}, w_{2}\right), 0\right)
$$

with $|v|=\left|w_{1}\right|=3,\left|w_{2}\right|=5, D w_{1}=0$ and $D w_{2}=v w_{1}$. Let $L\left(\mathbb{C} P^{2}\right)=L\left(B \cup_{\alpha} e^{4}\right)=\left(\mathbb{L}\left(u_{1}, u_{2}\right), \partial\right)$ with $\left|u_{1}\right|=1,\left|u_{2}\right|=3, \partial u_{1}=0$ and $\partial u_{2}=\left[u_{1}, u_{1}\right]$ [25]. Let

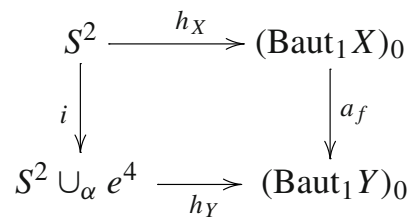

be a commutative diagram given by the DGL-model

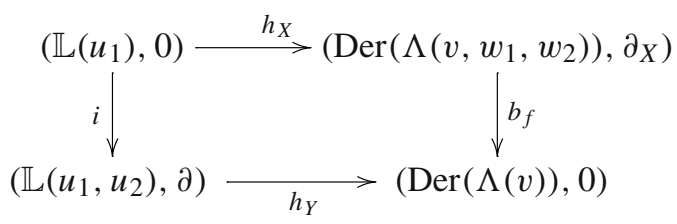

by $h_{X}\left(u_{1}\right)=h_{Y}\left(u_{1}\right)=0$ and $h_{Y}\left(u_{2}\right)=(v, 1)$. Then $\mathcal{O}_{\alpha}\left(h_{X}, h_{Y}\right) \neq 0$ in $H_{2}\left(\operatorname{Der}\left(\Lambda\left(w_{1}, w_{2}\right), \Lambda\left(v, w_{1}, w_{2}\right)\right)\right.$ since

$$
\tau h_{Y}\left(u_{2}\right)=\partial_{X}(v, 1)=\left(w_{2}, w_{1}\right) \nsim 0=h_{X}^{\prime \prime}\left(\left[u_{1}, u_{1}\right]\right)=h_{X}^{\prime \prime}\left(\partial_{\alpha}\left(u_{2}\right)\right) .
$$

Thus $h_{Y}: \mathbb{C} P^{2} \rightarrow\left(\text { Baut }_{1} Y\right)_{0}$ cannot lift to $h: \mathbb{C} P^{2} \rightarrow\left(\text { Baut }_{1} X\right)_{0}$. Note that $h_{Y}$ is extended to $\mathbb{C} P^{\infty} \rightarrow\left(\text { Baut }_{1} Y\right)_{0}$. Since $B S^{1}=\mathbb{C} P^{\infty}$, we obtain that any free $S^{1}$-action on $Y$ cannot lift to $X$.

\section{An application to lifting actions}

Let $B G$ and $E G$ be the classifying space and the universal space of a compact connected Lie group $G$ of rank $G=r$, respectively. If $G$ acts on a space $Y$ by $\mu: G \times Y \rightarrow Y$, there is the Borel fibration

$$
Y \stackrel{i}{\rightarrow} E G \times{ }_{G}^{\mu} Y \rightarrow B G
$$

where the Borel space $E G \times{ }_{G}^{\mu} Y$ (or simply $E G \times{ }_{G} Y$ ) is the orbit space of the diagonal action $g(e, y)=$ $\left(e g^{-1}, g y\right)$ on the product $E G \times Y$. It is rationally given by the KS extension (model)

$$
\left(\mathbb{Q}\left[t_{1}, \ldots, t_{r}\right], 0\right) \rightarrow\left(\mathbb{Q}\left[t_{1}, \ldots, t_{r}\right] \otimes \Lambda V, D_{\mu}\right) \rightarrow(\Lambda V, d)=M(Y) \quad(*),
$$

where $\left|t_{i}\right|$ are even for $i=1, \ldots, r, D_{\mu}\left(t_{i}\right)=0$ and $D_{\mu}(v) \equiv d(v)$ modulo the ideal $\left(t_{1}, \ldots, t_{r}\right)$ for $v \in V$.

Recall the lifting theorem of D. H. Gottlieb:

Theorem 5.1 [11, Theorem 1] Let a topological group $G$ acts on a space $Y$. A fibration $X \stackrel{f}{\rightarrow} Y$ is fibre homotopy equivalent to a $G$-fibration if and only if it is fibre homotopy equivalent to the pull-back of a fibration over $E G \times_{G} Y$ induced by the inclusion $i: Y \rightarrow E G \times_{G} Y$.

Proof of Theorem 1.10. Let $h_{Y}: B G \rightarrow\left(\text { Baut }_{1} Y\right)_{0}$ be the rationalization of the classifying map of the Borel fibration $Y \stackrel{i}{\rightarrow} E G \times{ }_{G}^{\mu} Y \rightarrow B G$ of the action $\mu: G \times Y \rightarrow Y$. Let $B^{n}$ be the n-skeleton of a CW complex $B$. From Theorem 1.8 , there is a lift $h_{X}^{\alpha}$ such that

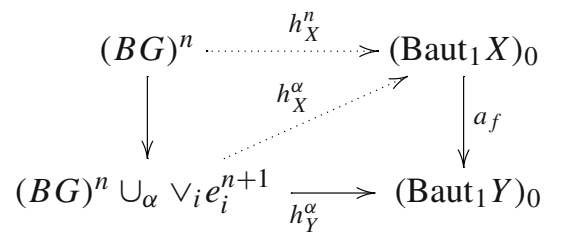


is commutative for all $n$ and attaching $\alpha$ since $\mathcal{O}_{\alpha}\left(h_{X}^{n}, h_{Y}^{\alpha}\right)=0$. Indeed, $\pi_{\text {odd }}\left(\operatorname{Baut}_{1} f\right)_{\mathbb{Q}}=0$ and $L(B G)$ is oddly graded since $H^{*}(B G ; \mathbb{Q})$ is evenly graded. Thus, we have the commutative diagram:

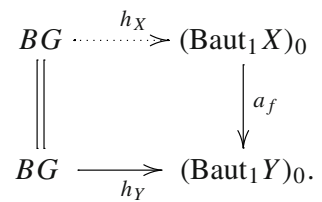

From Theorem 2.6, there is a commutative diagram:

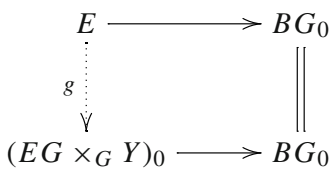

for some space $E$. Let $g^{\prime}: E^{\prime} \rightarrow E G \times_{G} Y$ be the pull-back of $g$ by the rationalization $l_{0}$ and $f^{\prime}: X^{\prime} \rightarrow Y$ be the pull-back of $g^{\prime}$ by $i$

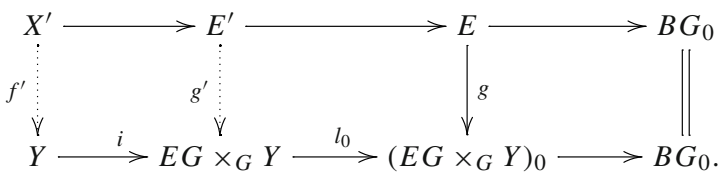

Notice that the model is given by the DGA-commutative diagram:

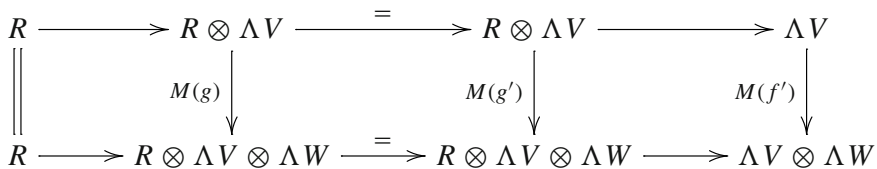

for $R:=H^{*}(B G ; \mathbb{Q})=\mathbb{Q}\left[t_{1}, \ldots, t_{r}\right]$. Notice that the third square is given by the push-out [7, Proposition 15.8]. Thus, from Theorem 5.1, we obtain the commutative diagram

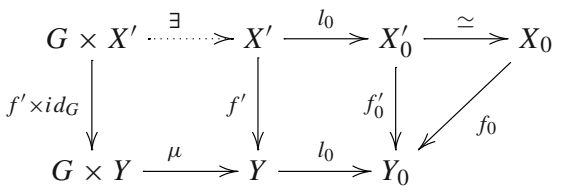

since $M\left(X^{\prime}\right) \cong \Lambda V \otimes \Lambda W=M(X)$.

If the $r$-torus $T^{r}$ acts on a space $Y,\left|t_{1}\right|=\cdots=\left|t_{r}\right|=2$ in $(*)$.

Proposition 5.2 [13, Proposition 4.2] Suppose that $Y$ is a simply connected $C W$-complex with $\operatorname{dim} H^{*}(Y ; \mathbb{Q})<$ $\infty$. Put $M(Y)=(\Lambda V, d)$. Then $r_{0}(Y) \geq r$ if and only if there is a KS extension ( $*$ ) satisfying $\operatorname{dim} H^{*}\left(\mathbb{Q}\left[t_{1}, \ldots, t_{r}\right] \otimes \wedge V, D\right)<\infty$. Moreover, if $r_{0}(Y) \geq r$, then $T^{r}$ acts freely on a finite complex $Y^{\prime}$ that has the same rational homotopy type as $Y$ and $M\left(E T^{r} \times T^{r} Y^{\prime}\right) \cong\left(\mathbb{Q}\left[t_{1}, \ldots, t_{r}\right] \otimes \wedge V, D\right)$.

Proof of Corollary 1.12. Let $r_{0}(Y)=r$. Notice that $L\left(B T^{r}\right)$ is oddly generated since $H^{*}\left(B T^{r} ; \mathbb{Q}\right)=$ $\mathbb{Q}\left[t_{1}, \ldots, t_{r}\right]$. Since $\pi_{\text {odd }}\left(\text { Baut }_{1} f\right)_{\mathbb{Q}}=0$, there exists a lift $\left(B T^{r}\right)_{0} \rightarrow\left(\text { Baut }_{1} X\right)_{0}$ from Theorem 1.8. Then we have the homotopy commutative diagram:

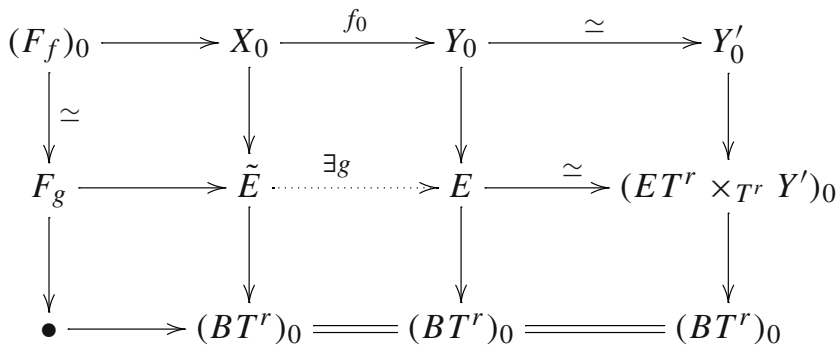


from Theorem 2.6. Here $\bullet$ is the one point space. We have $\operatorname{dim} H^{*}(\tilde{E} ; \mathbb{Q})<\infty \operatorname{since} \operatorname{dim} H^{*}\left(F_{g} ; \mathbb{Q}\right)<\infty$ and $\operatorname{dim} H^{*}(E ; \mathbb{Q})=\operatorname{dim} H^{*}\left(E T^{r} \times T^{r} Y ; \mathbb{Q}\right)<\infty$ for the fibration $F_{g} \rightarrow \tilde{E} \rightarrow E$. Thus there is a free $T^{r}$-action on $X^{\prime}$ with $X_{0}^{\prime} \simeq X_{0}$ and $\tilde{E} \simeq\left(E T^{r} \times_{T^{r}} X^{\prime}\right)_{0}$ from Proposition 5.2. Thus, we have $r_{0}(X) \geq r$.

Example 5.3 Let $S^{5} \rightarrow X \stackrel{f}{\rightarrow} Y$ be a rationally non-trivial fibration given by the model

$$
\left(\Lambda V, d_{Y}\right)=\left(\Lambda\left(v_{1}, v_{2}, v_{3}, v_{4}, v_{5}\right), d_{Y}\right) \rightarrow\left(\Lambda\left(v_{1}, v_{2}, v_{3}, v_{4}, v_{5}, w\right), D\right)
$$

with $\left|v_{1}\right|=\left|v_{2}\right|=2,\left|v_{3}\right|=\left|v_{4}\right|=\left|v_{5}\right|=|w|=5, d_{Y}\left(v_{1}\right)=d_{Y}\left(v_{2}\right)=0, d_{Y}\left(v_{3}\right)=v_{1}^{3}, d_{Y}\left(v_{4}\right)=v_{1}^{2} v_{2}$, $d_{Y}\left(v_{5}\right)=v_{2}^{3}$ and $D(w)=v_{1} v_{2}^{2}$. Then

$$
\pi_{\text {odd }}\left(\operatorname{Baut}_{1} f\right)_{\mathbb{Q}} \cong H_{\text {even }}\left(\operatorname{Der}_{\Lambda V}(\Lambda V \otimes \Lambda(w))\right)=0
$$

since there is no element of odd-degree $<5$ in $\Lambda V$. Therefore, $r_{0}(Y) \leq r_{0}(X)$. Indeed, we can directly check that $r_{0}(Y)=1$ and $r_{0}(X)=2$.

On the other hand, let $S^{5} \rightarrow X \stackrel{f}{\rightarrow} Y=S^{3} \times S^{3}$ be a rationally non-trivial fibration. Then the model is given by

$$
\left(\Lambda\left(v_{1}, v_{2}\right), 0\right) \rightarrow\left(\Lambda\left(v_{1}, v_{2}, w\right), D\right)
$$

with $\left|v_{1}\right|=\left|v_{2}\right|=3,|w|=5$, and $D(w)=v_{1} v_{2}$. Then

$$
\pi_{3}\left(\operatorname{Baut}_{1} f\right)_{\mathbb{Q}} \cong H_{2}\left(\operatorname{Der}_{\Lambda V}(\Lambda V \otimes \Lambda(w))\right)=\mathbb{Q}\left\{\left(w, v_{1}\right)\right\} \oplus \mathbb{Q}\left\{\left(w, v_{2}\right)\right\} \neq 0
$$

and $r_{0}(Y)=2>1=r_{0}(X)$.

Acknowledgements The author is supported by JSPS KAKENHI Grant Number JP20K03591.

Open Access This article is licensed under a Creative Commons Attribution 4.0 International License, which permits use, sharing, adaptation, distribution and reproduction in any medium or format, as long as you give appropriate credit to the original author(s) and the source, provide a link to the Creative Commons licence, and indicate if changes were made. The images or other third party material in this article are included in the article's Creative Commons licence, unless indicated otherwise in a credit line to the material. If material is not included in the article's Creative Commons licence and your intended use is not permitted by statutory regulation or exceeds the permitted use, you will need to obtain permission directly from the copyright holder. To view a copy of this licence, visit http://creativecommons.org/licenses/by/4.0/.

\section{References}

1. Allday, C.; Puppe, V.: Cohomological Methods in Transfomation Groups, vol. 32. Cambridge Univ. Press, Cambridge (1993)

2. Allaud, G.: On the classification of fiber spaces. Math. Z. 92, 110-125 (1966)

3. Booth, P.; Heath, P.; Morgan, C.; Piccinini, R.: H-spaces of self-equivalences of fibrations and bundles. Proc. London Math. (3) 49, 111-127 (1984)

4. Buijs, U.; Smith, S.B.: Rational homotopy type of the classifying space for fiberwise self-equivalenvces. Proc. AMS 141, 2153-2167 (2013)

5. Dold, A.; Lashof, R.: Principal quasi-fibrations and fibre homotopy equivalence of bundles. Ill. J. Math. 3, 285-305 (1959)

6. Félix, Y.; Halperin, S.: Rational LS category and its applications. Trans. AMS 273, 1-38 (1982)

7. Félix, Y.; Halperin, S.; Thomas, J.C.: Rational Homotopy Theory, Graduate Texts in Mathematics 205. Springer, Berlin (2001)

8. Félix, Y.; Lupton, G.; Smith, S.B.: The rational homotopy type of the space of self-equivalences of a fibration. Homol. Homotopy Appl. 12, 371-400 (2010)

9. Félix, Y.; Oprea, J.; Tanré, D.: Algebraic Models in Geometry, vol. 17. Oxford G.T.M, Oxford (2008)

10. Gatsinzi, J.B.: LS-category of classifying spaces. Bull. Belg. Math. Soc. Simon Stevin 2(2), 121-126 (1995)

11. Gottlieb, D.H.: Lifting Actions in Fibrations, vol. 657, pp. 217-254. Springer L.N.M, Berlin (1978)

12. Halperin, S.: Finiteness in the minimal models of Sullivan. Trans. AMS 230, 173-199 (1977)

13. Halperin, S.: Rational Homotopy and Torus Actions, London Math. Soc. Lecture Note Series, vol. 93, pp. $293-306$. Cambridge Univ. Press, Cambridge (1985)

14. Hilton, P.; Mislin, G.; Roitberg, J.: Localization of nilpotent groups and spaces. North Holland Math. Stud. 15, 1-156 (1975)

15. Jessup, B.; Lupton, G.: Free torus actions and two-stage spaces. Math. Proc. Camb. Phil. Soc. 137, 191-207 (2004)

16. Lupton, G.: Note on a Conjecture of Stephen Halperin, pp. 148-163. Springer L.N.M, Berlin (1990)

17. Peter May, J.: Fibrewise localization and completion. Trans. Am. Math. Soc 258, 127-146 (1980)

18. Meier, W.: Rational universal fibrations and flag manifolds. Math. Ann. 258, 329-340 (1982)

19. Nishinobu, H.; Yamaguchi, T.: Sullivan minimal models of classifying spaces for non-formal spaces of small rank. Topol. Appl. 196, 290-307 (2015)

20. Salvatore, P.: Rational homotopy nilpotency of self-equivalences. Topol. Appl. 77, 37-50 (1997) 
21. Shiga, H.; Tezuka, M.: Rational fibrations, homogeneous spaces with positive Euler characteristics and Jacobians. Ann. Inst. Fourier (Grenoble) 37, 81-106 (1987)

22. Smith, S. B.: The classifying space for fibrations and rational homotopy theory. In: The 5th GeToPhyMa Summer School on "Rational Homotopy Theory and its Interactions" in Rabat (2016)

23. Stasheff, J.: A classification theorem for fibre spaces. Topology 2, 239-246 (1963)

24. Sullivan, D.: Infinitesimal computations in topology. IHES 47, 269-331 (1978)

25. Tanré, D.: Homotopie Rationnelle: Modèles de Chen, Quillen, Sullivan. Lecture Note in Math, vol. 1025. Springer, Berlin (1983)

26. Thomas, J.C.: Rational homotopy of Serre fibrations. Ann. Inst. Fourier 331, 71-90 (1981)

Publisher's Note Springer Nature remains neutral with regard to jurisdictional claims in published maps and institutional affiliations. 